\section{Clinical reasoning in canine cervical hyperaesthesia: which presenting factors are important?}

\section{Nick Grapes, Steven De Decker, Rowena Packer}

Royal Veterinary College, London, United Kingdom

\section{OBJECTIVES}

To identify parameters from the history, presentation, physical and neurological examination of dogs presenting with cervical hyperaesthesia that were statistically associated with the underlying disease diagnosis.

\section{METHODS}

A retrospective study was performed containing 385 dogs presenting with cervical hyperaesthesia as the primary reason for clinical presentation between January 2010 and October 2018. Only cases presenting for cervical hyperaesthesia were included, while those presenting for others reasons with neck pain as a secondary problem were excluded.
Univariate analysis of variables (breed, age, weight, onset, progression, asymmetry, neuroanatomical localisation, haematology changes and pyrexia) were performed and variables with $\mathrm{P}<0.30$ were retained in a multinomial logistic regression model. Non-normally distributed continuous variables were analysed with a Kruskal-Wallis test.

\section{RESULTS}

$95.3 \%$ of cervical hyperaesthesia presentations were represented by only 8 conditions which included intervertebral disc extrusion (IVDE) ( $n=121)$, steroid-responsive meningitis arteritis (SRMA) $(n=100)$, syringomyelia (SM) $(n=63)$, intervertebral disc protrusion (IVDP) $(n=49)$, neoplasia ( $n=11$ ), cervical spondylomyelopathy (CSM) ( $n=10)$, meningitis of unknown aetiology (MUA) $(n=8)$ and immune-mediated polyarthritis (IMPA) $(n=5)$. Younger age, pyrexia and haematology changes were associated with a diagnosis of SRMA (all $p<0.001$ ). Neoplasia was common in older dogs $(p<0.001)$. Breed associations were evident with $81.4 \%(n=35)$ of French Bulldogs diagnosed with IVDE and $84.1 \%(n=53)$ of Cavalier King Charles Spaniels diagnosed with syringomyelia.

\section{STATEMENT (CONCLUSIONS)}

Easy-to-recognise clinical features can be used to identify the most likely differential diagnosis for dogs presenting with cervical hyperaesthesia. This information can be implemented by veterinary surgeons evaluating dogs with this clinical presentation.
Thoracic vertebral canal stenosis in screw-tailed brachycephalic dog breeds

\section{Alessandro Conte ${ }^{1}$, Marco Bernardini ${ }^{2}$, Steven De Decker ${ }^{3}$, Cristoforo Ricco4, Sebastien Behr', Daniel Sanchez-Masian", Giunio Bruto Cherubini', Luisa De Risio7, Rodrigo Gutierrez-Quintana ${ }^{8}$}

1 Animal Health Trust, Newmarket, United Kingdom 2 Ospedale Veterinario 'I Portoni Rossi', Zola Pedrosa, Italy 3 Royal Veterinary College - RVC, London, United Kingdom 4 Willows Veterinary Centre \& Referral Service, Solihull, United Kingdom

5 University of Liverpool, Liverpool, United Kingdom

6 Dick White Referrals, Six Mile Bottom, United Kingdom

7 Animal Health Trust, Newmarket, United Kingdom

8 Small Animal Hospital - University of Glasgow, Glasgow, United Kingdom

\section{OBJECTIVES}

To 1) describe clinical and imaging features of thoracic vertebral canal stenosis secondary to hypertrophy of the vertebral lamina and articular processes in screw-tailed brachycephalic dogs, to 2) evaluate its prevalence and to 3 ) determine if the degree of stenosis is associated with neurological signs.

\section{METHODS}

Retrospective multicentre study. Clinical records of screw-tailed brachycephalic dogs (French bulldogs, English bulldogs, Boston terriers and pugs) presented to seven institutions were reviewed. Twelve dogs with neurological deficits secondary to thoracic vertebral canal stenosis diagnosed on MRI were identified (Group 1). Signalment, clinical signs, imaging findings and treatment were recorded. One hundred and twenty-five neurologically normal dogs of the same breeds underwent CT imaging of the thoracic vertebral column for other medical reasons (Group 2). Cross-sectional measurements at the vertebral canal stenosis and immediately cranially were used to calculate a stenotic ratio. Both groups were compared using Mann-Whitney $U$ test $(p<0.05)$.

\section{RESULTS}

Group 1 consisted of three French bulldogs, eight English bulldogs and one pug. Nine were males. All dogs were 


\section{Oral presentations}

paraparetic on presentation; one was non-ambulatory. Twenty-three stenotic regions were identified with the most common region being T4-T5. Three dogs were treated surgically and all had a good long-term outcome. In Group 2, 33/175 dogs had at least one stenotic region with the most common being T2-T3. The degree of the stenosis was significantly higher in Group 1 ( $p=.01928)$.

\section{STATEMENT (CONCLUSIONS)}

Thoracic vertebral canal stenosis can affect screwtailed brachycephalic breeds. The vertebral canal in neurologically-affected screw-tailed brachycephalic dogs was $21.2 \%$ smaller compared to non-neurologically affected dogs.

\section{Assessment of quality of life in untreated mild idiopathic canine epilepsy}

\section{Lara Baptista ${ }^{1}$, Laura Barlow ${ }^{1}$, Lisa Alves ${ }^{2}$}

1 University of Cambridge, Cambridge, United Kingdom

2 University of Cambridge, Cambridge, United Kingdom

\section{OBJECTIVES}

To assess and compare the quality of life of a population of untreated dogs with mild idiopathic epilepsy versus a control group medicated with anti-epileptic drugs.

\section{METHODS}

Cross-sectional study. Two online questionnaires were developed for dogs receiving treatment and dogs untreated, and included quality of life associated questions. Carers were contacted by email and data acquired from 2009 to 2017. The results were analysed using Microsoft Excel ${ }^{\circledast}$ and Graphpad Software.

\section{RESULTS}

A total of 41 surveys were sent, with 26 replies for the control group and 7 for the untreated group and included 5 themes: "Abnormal behaviours", "Carers' quality of life"; "Dog's quality of life" and "Acceptability and progression of seizure frequency and severity". The untreated dogs did not show progression of seizure frequency or severity. There were statistically significant differences between the two groups in the quality of life of both the dogs and the carers. Epilepsy did not seem to impact the lives of carers in the non-medicated group. Equally, untreated dogs were perceived to have higher quality of life rates when compared to the medicated group.

\section{STATEMENT (CONCLUSIONS)}

Untreated patients with mild idiopathic epilepsy displayed higher quality of life ratings, as well as their carers, when compared to the medicated group. Moreover, there was no progression of disease in either frequency or severity, which contrasts with the accepted definition of epilepsy as a progressive condition, suggesting that in some patients there may be no disease progression. 